\section{Central Sulcus Focal Subarachnoid Hemorrhage in the Elderly: Cerebral Amyloid Angiopathy Is the Most \\ Frequent Cause}

With great interest I read the letter by Chandra et $\mathrm{al}^{1}$ referring to a review article by Cuvinciuc et al. ${ }^{2}$

Chandra et al ${ }^{1}$ present a 70 -year-old male patient with a focal subarachnoid hemorrhage (fSAH) in the left central sulcus. They conclude that an $80 \%$ stenosis of the left internal carotid artery (ICA), resulting in a shift of the watershed between the middle cerebral artery and the posterior cerebral artery territories toward the central sulcus, was responsible for the fSAH. Undoubtedly, the potential pathomechanism proposed by the authors is of interest; however, it is not at all proved in this case. We would like to draw attention to an alternative cause of this fSAH, namely cerebral amyloid angiopathy (CAA).

There is more and more evidence that CAA is the most important cause of nontraumatic fSAHs in older patients. ${ }^{3,4}$ fSAHs in patients with CAA most often affect the central sulcus, and patients typically present with transient ischemic attack (TIA)-like symptoms. ${ }^{3,5}$ According to the authors, CAA has been excluded by MR imaging in their case. I conclude that they found no additional typical MR imaging signs of CAA, such as old micro- or macrobleeds. However, several studies have found that $\mathrm{SSAH}$ or superficial siderosis as a residue of fSAH can be the only pathologic MR imaging finding in patients with histologically proved CAA. ${ }^{5}$ We propose that the coincidence of ICA stenosis and fSAH could be incidental.

On the basis of the available data in the literature and taking into account the advanced age of the patient (70 years), the localization of the fSAH (in the central sulcus), and the clinical presentation with "recurrent events" of "transient aphasia and dysarthria, along with right face and arm numbness," we regard CAA as the major differential diagnosis.

The consideration of CAA as the underlying cause in older patients with TIA-like symptoms and fSAH in the central sulcus is not only of academic interest, it also has a great clinical impact on patient management, especially in light of the risk of hemorrhagic complications of antiplatelet and anticoagulation therapy.

\section{References}

1. Chandra RV, Leslie-Mazwi TM, Oh D, et al. Extracranial internal carotid artery stenosis as a cause of cortical subarachnoid hemorrhage. AJNR Am J Neuroradiol 2011;32:E51-E52. Epub 2011 Feb 24

2. Cuvinciuc V, Viguier A, Calviere L, et al. Isolated acute nontraumatic cortica subarachnoid hemorrhage. AJNR Am J Neuroradiol 2010;31:1355-62. Epub 2010 Jan 21

3. Kumar N, Goddeau RP Jr, Selim MH, et al. Atraumatic convexal subarachnoid hemorrhage: clinical presentation, imaging patterns, and etiologies. Neurology 2010;74:893-99

4. Raposo N, Viguier A, Cuvinciuc V, et al. Cortical subarachnoid haemorrhage in the elderly: a recurrent event probably related to cerebral amyloid angiopathy. Eur J Neurol 2011;18:597-603. Epub 2010 Oct 6

5. Linn J, Halpin A, Demaerel P, et al. Prevalence of superficial siderosis in patients with cerebral amyloid angiopathy. Neurology 2010;74:1346-50

J. Linn

Department of Neuroradiology University Hospital Munich Munich, Germany

http://dx.doi.org/10.3174/ajnr.A2647 